\title{
Nivel de conocimiento acerca del dengue en los habitantes del Barrio Loma Pyta de la Ciudad de Asunción ${ }^{6}$ Level of knowledge about dengue in the inhabitants of Barrio Loma Pyta of the City of Asuncion
}

\author{
Liz Ozorio; Jesús Cantero; Jessica Isasi; Sonia Cáceres; Paola Parra; Pamela \\ Jara; Adolfo Montiel; Lilian Gamarra ${ }^{7}$; Maribel Acosta ${ }^{8}$; Claudia Centurión ${ }^{9}$;
} Gerardo Picón ${ }^{10}$

\section{DOI: https://doi.org/10.36003/Rev.investig.cient.tecnol.V2N2(2018)2}

\section{RESUMEN}

En las últimas décadas la incidencia de dengue en el mundo ha aumentado. Según estimaciones, se producen 390 millones de infecciones por dengue anualmente. En Paraguay, es considerada una enfermedad endémica desde el 2009. El único método para su prevención consiste en luchar contra la transmisión vectorial, es allí donde la educación juega un papel fundamental. El objetivo fue determinar el conocimiento sobre el dengue de los habitantes de la comunidad de Loma Pytá de la ciudad Asunción. El enfoque del estudio fue cuantitativo, diseño no experimental de tipo descriptivo, la técnica utilizada fue la entrevista y el instrumento una encuesta elaborada previamente según las variables de estudio. La población estuvo compuesta por familias que habitan en tres manzanas contiguas del Barrio Loma Pytá, el muestreo fue no probabilístico por conveniencia, la muestra estuvo constituida por 41 habitantes que cumplieron los criterios de inclusión y fueron encuestados en sus hogares. Como resultado se tuvo que el 58,5\% (24) de los entrevistados fueron del sexo femenino, el 39\%(16) tenían entre 18 a 23 años. Con respecto al conocimiento el 56\%(23) no conoce sobre el lugar de criadero y el 58\% (24) desconoce los síntomas. También, el 60\%(25) respondió que conoce sobre casos de dengue en los últimos tres meses. Se concluye la necesidad de reforzar los conocimientos de los pobladores del Barrio Loma Pytá sobre identificación de criaderos y síntomas del dengue. Se sugiere que estos temas sean ejes centrales de campañas de concientización y educación.

Palabras clave: Dengue; Enfermedades transmisibles emergentes; Conocimiento. 


\section{ABSTRACT}

In recent decades the incidence of dengue in the world has increased. According to estimates, 390 million dengue infections occur annually. In Paraguay, it is considered an endemic disease since 2009. The only method for its prevention is to fight against vector transmission, this is where education plays a fundamental role. The objective was to determine the knowledge about dengue of the inhabitants of the community of Loma Pytá in the city of Asunción. The focus of the study was quantitative, non-experimental design of descriptive type, the technique used was the interview and the instrument a survey previously prepared according to the study variables. The population was composed of families living in three contiguous blocks of Barrio Loma Pytá, sampling was not probabilistic for convenience, the sample consisted of 41 inhabitants who met the inclusion criteria and were surveyed in their homes. As a result, 58.5\% (24) of the interviewees were female, $39 \%$ (16) were between 18 and 23 years old. With respect to knowledge, $56 \%$ (23) do not know about the breeding place and 58\% (24) do not know the symptoms. Also, $60 \%$ (25) responded that they know about cases of dengue in the last three months. The need to reinforce the knowledge of the inhabitants of Barrio Loma Pyta on identification of breeding sites and symptoms of dengue is concluded. It is suggested that these issues be central axes of awareness and education campaigns.

Keywords: Dengue; Emerging communicable diseases; Knowledge. 


\section{INTRODUCCIÓN}

En las últimas décadas la incidencia de dengue en el mundo ha aumentado. Según estimaciones, se producen 390 millones de infecciones por dengue anualmente. Los estados miembros de la Organización Mundial de la Salud (OMS) notifican sistemáticamente el número anual de casos, el número de casos notificados pasó de 2,2 millones en el 2010 a 3,2 millones en el 2015 (1).

El dengue es el nombre de una enfermedad infecciosa y de tipo epidémico, que se produce por el virus transmitido por los mosquitos Aedes Aegyptis y Aedes Albopictus, (2). Estos mosquitos suelen aparecer en regiones de climas tropicales, incubando sus huevos en aguas estancadas que pueden generar diversos signos y síntomas que pueden llegar a ser mortales (3).

El dengue es una enfermedad viral aguda que puede afectar a personas de cualquier edad, pero son más susceptibles los niños y los adultos mayores. Los mosquitos del dengue ponen sus huevos en depósitos de agua limpia como albercas, floreros de plantas acuáticas, llantas, baldes de agua y cualquier recipiente que está a la intemperie y que puede almacenar agua (4). Allí se desarrollan las larvas y después pasan a su forma adulta que es la que transmite el virus. El dengue nace de nuestros comportamientos. Si mantenemos nuestros patios limpios, si tenemos un adecuado uso de los desechos evitamos que se conviertan en potenciales reservorios de mosquitos (4).

En Paraguay, es considerada una enfermedad endémica desde el 2009. Según los datos del Ministerio de Salud Pública y Bienestar Social (2018) unos 1439 casos de Dengue fueron confirmados a nivel país hasta el 17 de febrero del 2018 y unos 2391 casos probables. Las estadísticas refieren un promedio de 3888 notificaciones en las últimas 3 semanas (5). El único método para su prevención consiste en luchar contra la transmisión vectorial, es allí donde la educación juega un papel fundamental (6).

La prevención o reducción de la transmisión del virus del dengue depende por completo de que se controlen los mosquitos vectores o se interrumpa el contacto entre estos y los seres humanos. Para controlar los mosquitos vectores, como los del dengue, la OMS promueve un enfoque estratégico, conocido como control integrado de vectores. Es un proceso racional de toma de decisiones para optimizar el uso de recursos en el control de los vectores. Sus objetivos son la mejora de la eficacia, la rentabilidad, la congruencia ecológica y la sostenibilidad (6). Las actividades para controlar la transmisión deben centrarse en los mosquitos Aedes Aegypti (u otros vectores, siempre que haya pruebas de que transmiten el dengue) en estadios inmaduros (huevo, larva y pupa) y en la etapa adulta en el interior de viviendas y espacios adyacentes. También se incluyen otros entornos donde se produce el contacto entre seres humanos y vectores, como escuelas, hospitales y centros de trabajo (7). Con base sobre el incremento considerable, se considera relevante proponer la siguiente interrogante: ¿Cuál es el nivel de conocimiento de los habitantes de la comunidad de Loma Pyta sobre el dengue?

El objetivo de esta investigación fue determinar el conocimiento sobre el dengue de los habitantes de la comunidad de Loma Pytá de la ciudad Asunción.

\section{MÉTODO}

El enfoque del estudio fue cuantitativo, diseño no experimental de tipo descriptivo, la técnica utilizada fue la entrevista y el instrumento una encuesta elaborada previamente según las variables de estudio. La población estuvo compuesta por familias que habitan en tres manzanas contiguas 
del Barrio Loma Pytá, el muestreo fue no probabilístico por conveniencia, la muestra estuvo constituida por 41 habitantes que cumplieron los criterios de inclusión y fueron encuestados en sus hogares. Criterios de inclusión. Personas residentes en la vivienda seleccionada que atendió la puerta y que manifestó su interés en participar en la encuesta, mayor de 18 años de edad. Criterios de exclusión. Personas que no tenían como mínimo 1 año de vivir en el barrio y menores de 17 años. Las variables a estudio fueron: sexo, edad, conocimientos sobre el dengue, de los signos y síntomas de la enfermedad, mecanismos de transmisión. Se utilizó el criterio de expertos para garantizar la validez por constructo y de contenido. Para la recolección de la información se consideró el deseo de voluntad de participación, se explicó la importancia del estudio, sus propósitos y perspectivas, manteniendo siempre los principios que rigen la ética investigativa.

Posteriormente los datos recolectados fueron insertados en planillas Excel y exportados al programa estadístico SPSS (Statical Package for the Social Sciences) para los análisis utilizando estadística descriptiva (frecuencias y porcentajes).

\section{RESULTADOS}

Como resultado se tuvo que el 58,5\% (24) de los entrevistados fueron del sexo femenino, el 39\%(16) tenían entre 18 a 23 años. Con respecto al conocimien- to el $56 \%(23)$ no conoce sobre el lugar de criadero y el $58 \%$ (24) desconoce los síntomas como se observa en el gráfico 1.

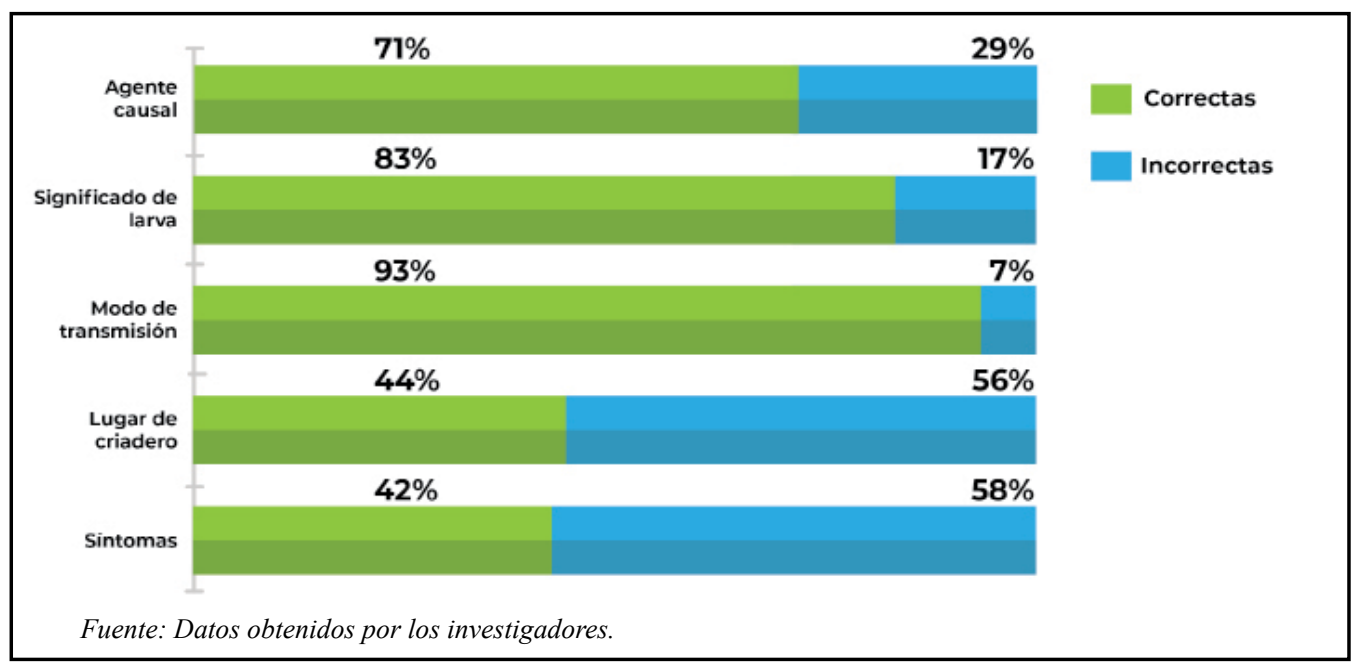

Gráfico 1. Distribución porcentual de habitantes según ítems de respuesta. Barrio Loma Pytá. Asunción. 2018. $n=41$. 


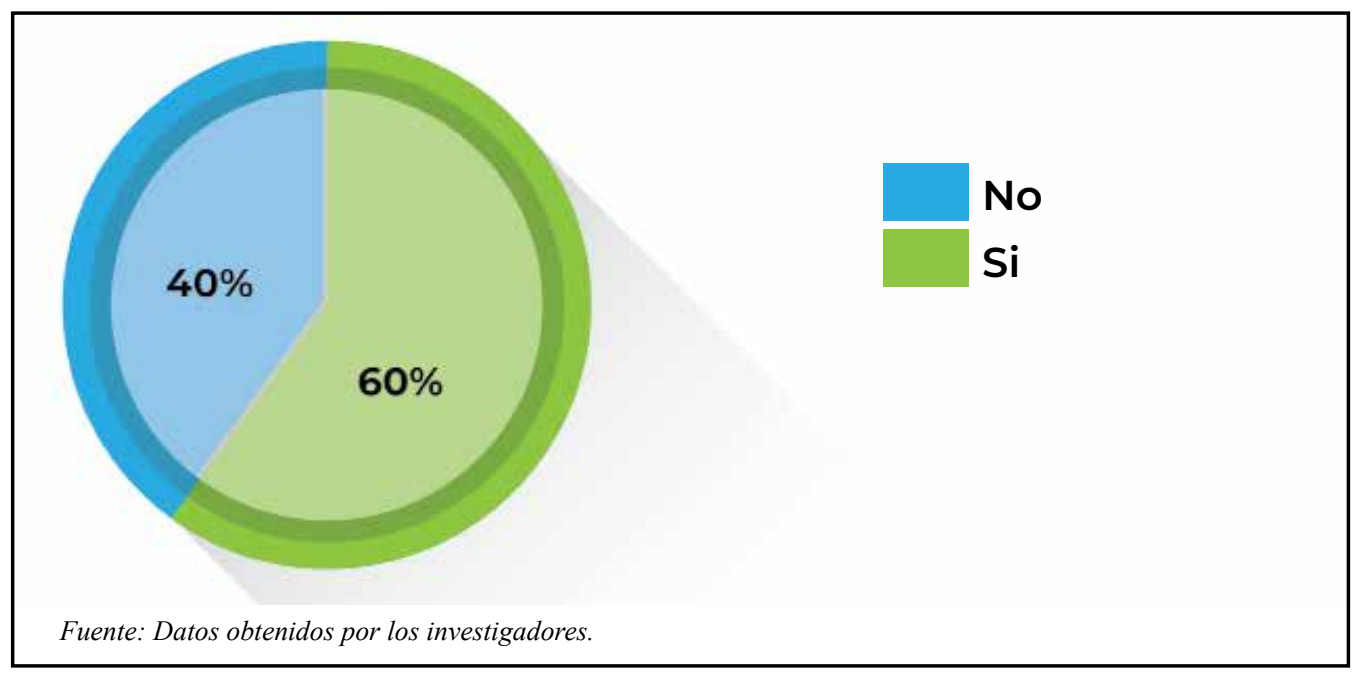

Gráfico 2. Distribución porcentual de habitantes según conocimiento sobre casos de dengue en los últimos 3 meses. Barrio Loma Pyta. Asunción. 2018. n=41.

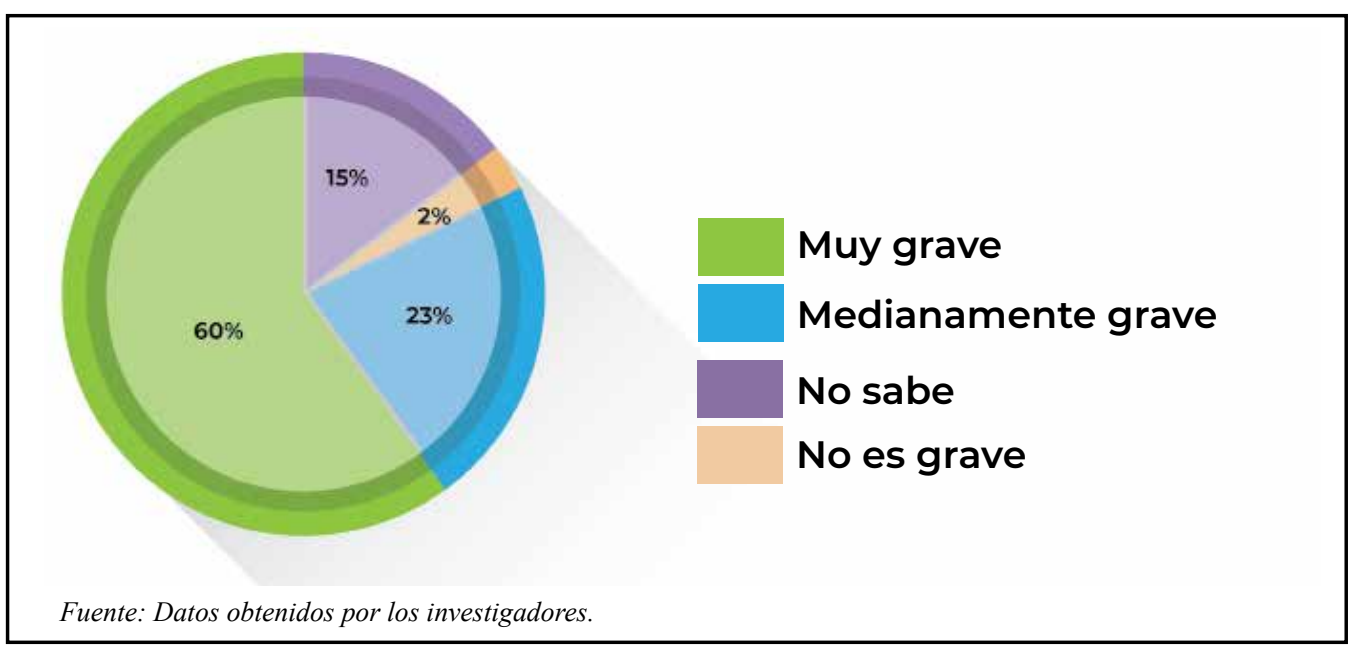

Gráfico $N^{\circ} 3$. Distribución porcentual de habitantes según percepción sobre la gravedad de la enfermedad del dengue. Barrio Loma Pyta. Asunción. 2018. $n=41$.

\section{DISCUSIÓN}

Los resultados de un estudio realizado en Venezuela, en el periodo comprendido de mayo a junio de 2007, para determinar el nivel de conocimientos sobre el dengue evidenciaron el alto desconocimiento de la población sobre la enfermedad y ausencia de información sobre los síntomas, y control frente a dengue. Se determinó mecanismo de adquisición del dengue, su agente transmisor y prevención (8). Otro estudio de tipo descriptivo similar se realizó en la región Piura de Perú para describir los conocimientos sobre transmisión, sintomatología, acciones de prevención 
que el conocimiento sobre algunos aspectos del dengue es aún limitado en la región Piura, siendo ésta la más endémica a nivel nacional (9).

Otra investigación realizada en Colombia, Yopal, Casanare tuvo como objetivo identificar conocimientos-actitudes-prácticas sobre dengue evidenció que los sujetos del estudio reconocen el dengue como una enfermedad e identifican claramente el nombre del vector e identifican los signos y síntomas de la enfermedad (10).

En Paraguay, una investigación realizada tuvo como objetivo determinar los cono- cimientos, actitudes y prácticas que tenían los pobladores del barrio San Pablo de la ciudad de Asunción y se estableció que la población estudiada poseía conocimientos adecuados con respecto a las características del dengue y el control de los mosquitos, escasa participación en actividades comunitarias, los conocimientos adecuados no necesariamente derivaban en prácticas preventivas (11). Este resultado es muy similar al encontrado en la comunidad de Loma Pyta, ya que los habitantes tenían conocimientos sobre el tema estudiado.

\section{CONCLUSIONES}

Se concluye la necesidad de reforzar los conocimientos de los pobladores del Barrio Loma Pytá sobre identificación de criaderos y síntomas del dengue. Se sugiere que estos temas sean ejes centrales de campañas de concientización y educación.

\section{BIBLIOGRAFÍA}

1. Organización Mundial de la Salud. Dengueydenguegrave.2018Disponibleen: https:/www.who.int/es/news-room/factsheets/detail/dengue-and-severe-dengue

2. Centros para el Control y la Prevención de Enfermedades. Enfermedades y afecciones: Dengue. Disponible en: https://www.cdc.gov/spanish/enfermedades/ dengue/hojadatos.html

3. TConsejo de Educación Inicial y Primaria. Aedes aegypti y Aedes albopictus . Disponible en: http://www.ceip.edu. uy/documentos/galerias/prensa/1243/pre_ aedes_aegypti.pdf

4. Ministerio de Salud y Protección Social. Dengue. Disponible en: https:// www.minsalud.gov.co/salud/Paginas/Dengue.aspx

5. Ministerio de Salud Pública $y$ Bienestar Social. Dengue: Amenaza de propagación sigue latente. Disponible en: https://www.mspbs.gov.py/portal/11089/ dengue-amenaza-de-propagacion-sigue-latente.html

6. Organización Mundial de la Salud. Lucha contra el dengue: estrategias de lucha antivectorial. Disponible en: https:// www.who.int/denguecontrol/control_strategies/es/

7. Organización Panamericana de la Salud. Nota descriptiva. Acerca del dengue. Disponible en: https://www.paho. org/par/index.php?option=com_content\&view $=$ article \&id=443: control-vectorial-aedes-aegypti-mediante-rociado-espacial\&Itemid $=258$.

8. Hoyos Rivera, A. Pérez Rodríguez, A. Nivel de conocimientos sobre el dengue en San Mateo, Anzoátegui, Venezuela. Rev Cubana Salud Pública [Internet]. 2009 Dic; 35( 4 ): 161-172. Disponible en: http:// 
scielo.sld.cu/scielo.php?script=sci_arttext\&pid=S0864-34662009000400015\&ln$\mathrm{g}=\mathrm{es}$.

9. Gutiérrez, C. Montenegro-Idrogo J. Conocimiento sobre dengue en una región endémica de Perú: Estudio de base poblacional. Acta méd. Peru [Internet]. 2017 Oct [citado 2019 Feb 27] ; 34( 4 ): 283288. Disponible en: http://www.scielo. org.pe/scielo.php?script=sci_arttext\&pi$\mathrm{d}=\mathrm{S} 1728-59172017000400005 \& \operatorname{lng}=$ es.

10. Criollo Fonseca, I.Z., Bernal Barón, A. Y. Castañeda Porras, O. Conocimientos, actitudes y prácticas sobre dengue, tras aplicación de estrategias de movilización social. Yopal-Casanare, Co- lombia, 2012 Revista Investigaciones Andina. No. 29 Vol. 16 - 150 p. Disponible en: http://www.scielo.org.co/pdf/inan/ v16n29/v16n29a02.pdf

11. Stela Benítez-Leite, M.L. Machi, E. Gibert K. Rivarola Conocimientos, actitudes y prácticas acerca del dengue en un barrio de Asunción. Revista Arch.argent. pediatr 2001; 99(5) Disponible en: https:// www.sap.org.ar/docs/publicaciones/archivosarg/2001/437.pdf 